\title{
ACCIONES PARA LA IMPLEMENTACIÓN DE PROCESOS INCLUSIVOS EN INSTITUCIONES DE EDUCACIÓN SUPERIOR ${ }^{1}$
}

\author{
Yhara Gutiérrez², Marilyn San Martín³
}

\begin{abstract}
RESUMEN
En nuestro país, el acceso inclusivo a la educación superior se rige por leyes y decretos que regulan los procedimientos, pero no entregan lineamientos específicos de cómo llevarlos a cabo. Este estudio, por tanto, busca develar las principales acciones para el ingreso y mantenimiento de estudiantes con discapacidad a la educación superior. Para esto se optó por una investigación cualitativa, a partir de un muestreo intencionado y entrevistas semiestructuradas en las que participaron ocho instituciones nacionales de enseñanza superior. Para el análisis de información se utilizó la técnica de teorización anclada. Los resultados fueron organizados en tres niveles de análisis: el primero, para describir procesos de admisión, programas de apoyo a la inclusión y accesibilidad; el segundo, para analizar la eficiencia de los programas de inclusión, y el tercero para realizar un análisis global, categorizando barreras y facilitadores para la inclusión. Los resultados permiten concluir que, no obstante los avances en la materia, aún se requiere que las instituciones de educación superior promuevan políticas institucionales que permitan a la comunidad educativa cambiar el paradigma inclusivo e institucionalizar los apoyos, considerando que no existe una fórmula única, ya que los apoyos dependerán de la cultura de cada institución.
\end{abstract}

Conceptos clave: inclusión, discapacidad, derecho a la educación.

\section{ACTIONS TO IMPLEMENT INCLUSIVE PROCESSES IN HIGHER EDUCATION INSTITUTIONS}

\section{ABSTRACT}

In Chile, access to higher education is governed by laws and decrees that regulate inclusive procedures, but do not provide specific guidelines on how to carry it out. This study seeks to reveal the main actions for admission and maintenance of students with disabilities in higher education. For this purpose, a qualitative investigation was carried out, based on intentional sampling and semi-structured interviews with the participation of 8 higher education national institutions. To analyze the information, the Grounded Theory technique was used. The results were organized into three levels of analysis: First, describing admission processes, programs to support inclusion and accessibility; second, analyzing the efficiency of inclusion programs; and finally, carrying out a global analysis, categorizing barriers or facilitators for inclusion. The results allowed us to

1 Este artículo está basado en una tesis para optar al grado de Magíster en Educación mención gestión inclusiva de la Universidad Santo Tomás (2019).

2 Universidad del Bío-Bío, Concepción, Chile. Contacto: ygutierrez@ubiobio.cl

3 Universidad del Bío-Bío, Concepción, Chile. Contacto: msanmartin@ubiobio.cl 
conclude that, despite the advances in the matter, higher education institutions are still required to promote institutional policies that affect the educational community in order to change the inclusive paradigm, and to institutionalize support, considering that there is no single formula, since support depends on the culture of each institution.

Key concepts: Inclusion, Disability, Right to Education. 


\section{Introducción}

La UNESCO (2008) establece que la tarea educativa no solo debe generar procesos de integración, sino también las condiciones que permitan contener y mantener en los sistemas educativos a todos los estudiantes. Asimismo, la actual normativa legal y la reforma educacional en Chile plantean una educación constituida sobre la base de la equidad, en la que todos los estudiantes tengan la posibilidad de una educación de calidad en igualdad de oportunidades. En este sentido, el modelo establecido en las nuevas políticas educacionales del país tiene como propósito identificar las dificultades de los estudiantes, con el objetivo de beneficiar el logro de los aprendizajes a partir de las competencias individuales y satisfacer estas necesidades en cualquier contexto educativo, buscando que las instituciones tengan un carácter inclusivo, es decir, que se hagan cargo de la diversidad de los estudiantes, asegurando que el sistema los favorezca a todos por igual, no considerando la diversidad como un obstáculo o problema sino como una realidad que complejiza y enriquece el proceso de enseñanza-aprendizaje (Lissi, Zuzulich, Salinas, Achiardi, Hojas y Pedrals, 2009). Por lo tanto, el gran desafío es dotar a las instituciones educativas y, especialmente a docentes y académicos, de las condiciones, herramientas pedagógicas, materiales y ayudas técnicas necesarias para favorecer el logro de aprendizajes de los estudiantes, su inclusión y, por ende, su participación en la sociedad.

Las cifras en Latinoamérica evidencian que la temátiva de inclusión en educación superior es escasa, además, hay insuficiente producción científica que entregue información acerca de los estudiantes en este nivel. No obstante, Chile es el país en América Latina donde se han realizado más investigaciones acerca de inclusión educativa en personas con discapacidad (Paz-Maldonado, 2020).

El Informe de la Organización Mundial de la Salud (OMS) de 2011 señala que el 15\% de la población mundial vive en condición de discapacidad. En 2016, en Chile, se publican los resultados del II Estudio Nacional de la Discapacidad, que informan de un $16,7 \%$ de personas en esta condición. Un 8,5\% de las personas con discapacidad se encuentra actualmente estudiando y, de ellas, un 
5,9\% posee enseñanza superior incompleta y un 9,1\% enseñanza superior completa (SENADIS, 2016).

En la actualidad, los derechos de personas en situación de discapacidad se respaldan con la Convención sobre los Derechos de las Personas con Discapacidad, convención que en nuestro país fue ratificada en 2008, y la Ley $\mathrm{N}^{\circ} 20.422$, que establece normas sobre igualdad de oportunidades e inclusión social de personas con discapacidad. Este marco normativo exige al Estado implementar medidas y acciones que aseguren el acceso universal, la equidad y la participación social de estas personas. (Ley No 20.422, 2010). Este cuerpo legal, en su artículo 3, hace referencia al Diseño Universal de Aprendizaje (DUA), que se fundamenta en las bases neurocientíficas de cómo las personas aprenden, siendo su objetivo diseñar y planificar estrategias en un escenario didáctico que sea accesible para todos (Lagos, 2019).

Consecuentemente, en mayo de 2018 se publicó la ley $\mathrm{N}^{\circ}$ 21.091, cuyo objetivo es fortalecer el aseguramiento de la calidad de la educación y determinar los requisitos que deben cumplir los planteles de educación superior para acceder al financiamiento institucional para la gratuidad, además de promover la inclusión de estudiantes, velando por la eliminación y prohibición de todas las formas de discriminación arbitraria. Asimismo, la normativa incita a la realización de ajustes razonables, entendidos como modificaciones y adaptaciones necesarias que permitan a las personas compensar alguna deficiencia que les impida el goce o ejercicio de todos los derechos humanos y libertades fundamentales (Castellanos y Quintana, 2019). Estos ajustes permitirían la inclusión y accesibilidad de las personas con discapacidad en procedimientos de admisión y demás aspectos de la educación superior.

El aspecto más visible en cuanto a accesibilidad se relaciona con espacios físicos y arquitectura de edificios en los que se encuentran insertas las instituciones de educación superior. En 2016 se publicó el Decreto Supremo No 50 , que actualiza sus normas a las disposiciones de la ley No 20.422 sobre Igualdad de Oportunidades e Inclusión Social de Personas con Discapacidad, y dispone que le 
corresponde al Ministerio de Vivienda y Urbanismo establecer, en la Ordenanza General de Urbanismo y Construcciones, las normas a las que deberán sujetarse las nuevas obras y edificaciones que ese artículo señala, así como las normas y condiciones para que las obras y edificaciones existentes se ajusten gradualmente a las nuevas exigencias de accesibilidad.

Conjuntamente, este Decreto regula la existencia de un Sistema de Acceso a Instituciones de Educación Superior, que respete la diversidad de talentos, capacidades y trayectorias previas de los estudiantes. El sistema debe contemplar procesos e instrumentos de acceso de aplicación general, considerando las particularidades de cada subsistema; además, permitir que las instituciones de educación superior desarrollen instrumentos específicos, complementarios a los generales, con el objeto de fomentar la equidad en el ingreso de estudiantes (Ley No 21.091, 2018). En este mismo sentido, la Ley Antidiscriminación, publicada en 2012, instaura un mecanismo judicial que regula el procedimiento cuando se cometa un acto de discriminación arbitraria, fundado en motivos que incluyen, entre otros, la discapacidad (Ley No 20.609, 2012).

En efecto, estas leyes, en conjunto, entregan las primeras directrices para otorgar igualdad de oportunidades a estudiantes que ingresan a la educación superior; no obstante, son las mismas instituciones las encargadas de crear y llevar a cabo acciones para dar cumplimiento a la normativa. Respecto del ingreso a la educación superior, existen dos realidades distintas: por una parte, las instituciones que reciben estudiantes a través de la Prueba de Selección Universitaria (PSU) y, por otra, aquellas que lo hacen con sus propios sistemas de admisión. Generalmente, las que lo hacen por PSU, además, han tenido sistemas de admisión especial para estudiantes en situación de discapacidad (EsD), con cupos y carreras limitadas. No obstante, el Departamento de Evaluación, Medición y Registro Educacional (DEMRE), con la finalidad de dar respuesta a la diversidad, amplía la accesibilidad de la Prueba de Selección Universitaria, realizando ajustes a la PSU. Esto permite que las personas en situación de discapacidad postulen a sus preferencias en igualdad de condiciones (DEMRE, 2019). 
Esta nueva perspectiva educativa, que tiene como finalidad el respeto y considera en el quehacer educativo la no homogenización, da paso a nuevos desafíos en educación para equiparar las oportunidades de todas las personas en situación de discapacidad. Para quienes trabajamos en el área educativa, existe un conjunto de inquietudes respecto de las acciones y prácticas que se llevan a cabo para erradicar la exclusión, ya que, para que el concepto de inclusión sea real, las buenas prácticas deben traspasar el límite de lo puramente educativo. Se requiere también de un cambio de mentalidad en la sociedad en general, puesto que, muchas veces, la exclusión social se genera producto de actitudes ante la diversidad racial, social, étnica, de género, de habilidades. Esto explica, en parte, la estrecha relación que existe entre inclusión y los derechos humanos (Guerra, 2006). La inquietud por responder a los requerimientos y necesidades de los EsD que estaban, están o estarán en la educación superior explica la creación de la Red de Educación Superior Inclusiva (RESI), organización sin fines de lucro en la que participan todas las instituciones de educación superior, públicas y privadas del país, con la intención de compartir procesos y buenas prácticas en lo referente a la inclusión de EsD; unificar criterios respecto de las decisiones y gestiones a realizar; intercambiar conocimientos, información y experiencia en torno a la promoción de la inclusión educacional y social de las personas en situación de discapacidad, e impulsar políticas públicas para garantizar los derechos de estos estudiantes (RESI, 2018).

Frente a lo expuesto, surge la interrogante respecto de las acciones que se llevan a cabo en las instituciones de educación superior para el cumplimiento de la Ley de Inclusión, las estrategias para el acceso al currículo, materiales curriculares y detección de barreras de acceso al aprendizaje y a la participación.

Por lo tanto, esta investigación busca contribuir a paliar la escasez de información e investigaciones respecto de inclusión y educación superior, y, más aún, de estrategias exitosas utilizadas en nuestro país. Adicionalmente, desde lo teórico, aportar al análisis documental y bibliográfico relacionado con el tema, contrastado con los aportes de la teoría o paradigmas que convergen a este tema de la 
diversidad en educación. Finalmente, desde un aspecto práctico, la información sistematizada servirá de insumo para identificar, describir y analizar las acciones adoptadas por las instituciones de educación superior, y podría ser la base para proponer nuevas e innovadoras estrategias que permitan el acceso, permanencia y egreso de los EsD que optan por continuar sus estudios superiores, permitiendo replicar experiencias y buenas práctica tanto de admisión y atención a la diversidad, como de acompañamiento durante el tiempo que los estudiantes se encuentren en sus instituciones.

\section{Metodología}

\subsection{Tipo de investigación, contexto y participantes}

Se escogió un diseño descriptivo y exploratorio con una metodología cualitativa y con enfoque de teorización anclada (Strauss, 1989, citado en Soler y Fernández, 2010). Se optó por este enfoque por su capacidad de precisar un fenómeno, entendiendo la acción desde la perspectiva de los actores. Se utilizó un muestreo por conveniencia, fundamentado en la proximidad y accesibilidad de los sujetos para el investigador (Otzen y Manterola, 2017).

Se realizó una convocatoria para participar en el estudio a las 11 instituciones de educación superior que han declarado contar con EsD y que pertenecen a RESI en la región del Biobío. Las cartas fueron enviadas a los decanatos y directorios para su aprobación.

La selección de los informantes se estableció de acuerdo con los siguientes criterios: universidades, centros de formación técnica e institutos de educación superior de la región del Biobío que participan activamente en la RESI. Finalmente, ocho instituciones accedieron a participar.

Posteriormente se realizó una entrevista a representantes de cada una de las instituciones, excepto de la Institución 2, que cuenta con dos programas de inclusión, cada uno con su respectivo representante en la RESI. 


\subsection{Técnica de recolección e información}

Se elaboró un guion con el propósito de responder a los objetivos propuestos en la investigación. El guion estuvo constituido por 24 preguntas extraídas del Índex para la Inclusión (Booth y Ainscow, 2015), además de seis preguntas de elaboración propia. De esta manera, el instrumento quedó constituido por dos ítems: uno con enfoque hacia los procesos de admisión y el otro relacionado con acciones desarrolladas para el acceso al currículo.

El guion fue sometido a juicio de expertos para su validación, con el propósito de obtener mayor objetividad y factibilidad de administración. Se contó con la colaboración de tres especialistas del área de educación. Las expertas realizaron comentarios y sugerencias, principalmente relacionadas con la redacción, y no hubo indicaciones que sugirieran eliminar preguntas. Con las observaciones y aportes de estas profesionales el instrumento fue modificado y mejorado para su aplicación. Se envió nuevamente a las juezas expertas quienes aprobaron las 30 preguntas.

Consecuentemente, este guion de preguntas se utilizó para la realización de la entrevista, con el fin de acceder en profundidad al propio lenguaje de los representantes de la RESI, a sus opiniones, vivencias, experiencias, creencias, ideas, prejuicios, códigos y estereotipos, y acerca de las acciones que ejecutan e implementan para promover la inclusión en educación superior. Además, esta técnica permitió al entrevistador señalar dudas, focalizar o profundizar aquellos temas que consideró pertinentes, y replantear ideas que necesitó aclarar con el objetivo de ahondar de acuerdo con el problema de investigación (Canales, 2006).

\subsection{Técnica de análisis}

La técnica de análisis de la información corresponde a la teorización anclada, realizada a partir del análisis del discurso, desde categorías teóricas que surgieron a partir de la transcripción de las 30 preguntas respondidas por los entrevistados. Estas preguntas fueron incorporadas al cuestionario y posteriormente anexadas al programa Atlas Ti 8.2 para su análisis y codificación. 
En esta etapa se identificaron las citas más relevantes y recurrentes, y se obtuvieron 295 citas. Posteriormente, se las agrupó de acuerdo con la temática que trataban y se las etiquetó manteniendo el propio lenguaje utilizado por los entrevistados. Cinco citas fueron eliminadas, porque no pertenecían a ninguna familia o grupo de códigos. Las 290 citas dieron lugar al procedimiento que, de acuerdo con Strauss y Corbin (en Giraldo, 2011), corresponde a la asignación de códigos en vivo, generándose 70 códigos.

Por último, con el análisis previo rigurosamente cumplido, los códigos fueron analizados según sus semejanzas y diferencias conceptuales, luego de lo cual se les organizó en torno a familias o grupos de códigos de acuerdo con la categoría o concepto a la que se referían. De esta etapa se desprendieron cuatro grupos de códigos o familias, los que fueron analizados, categorizados, subcategorizados y descritos en tres niveles de análisis: 


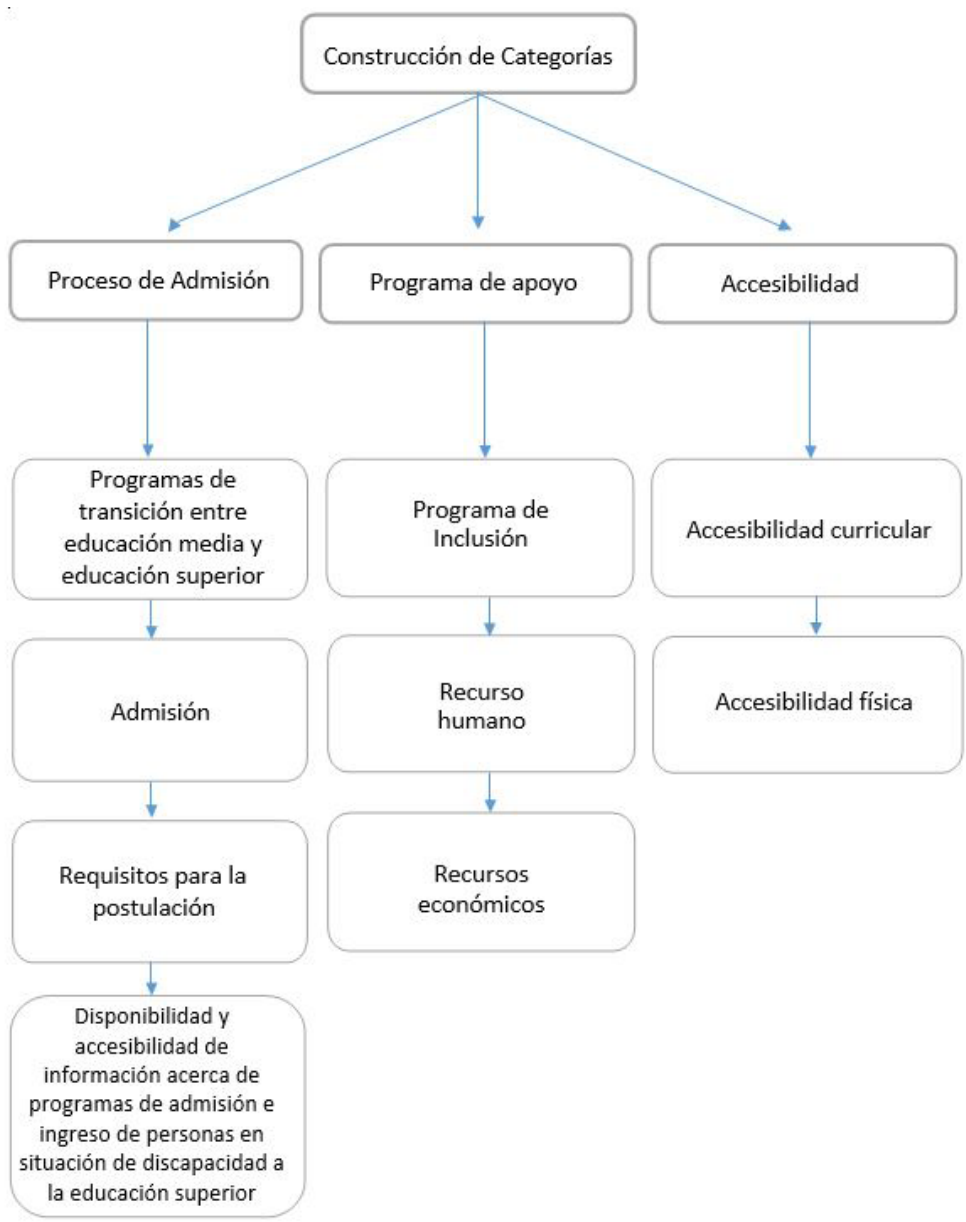

\section{Figura $N^{o} 1$}

Primer nivel de análisis 
294 ACCIONES PARA LA IMPLEMENTACIÓN DE PROCESOS INCLUSIVOS EN INSTITUCIONES DE EDUCACIÓN SUPERIOR - Y. Gutiérrez, M. San Martín

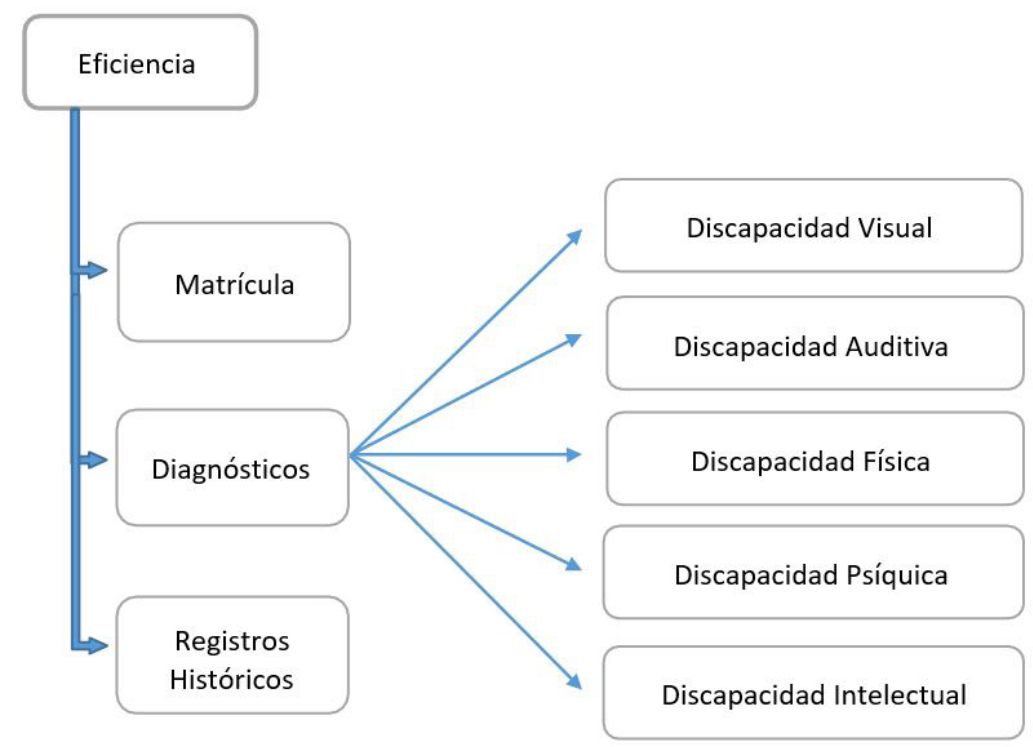

Figura $N^{\circ} 2$

Segundo nivel de análisis 

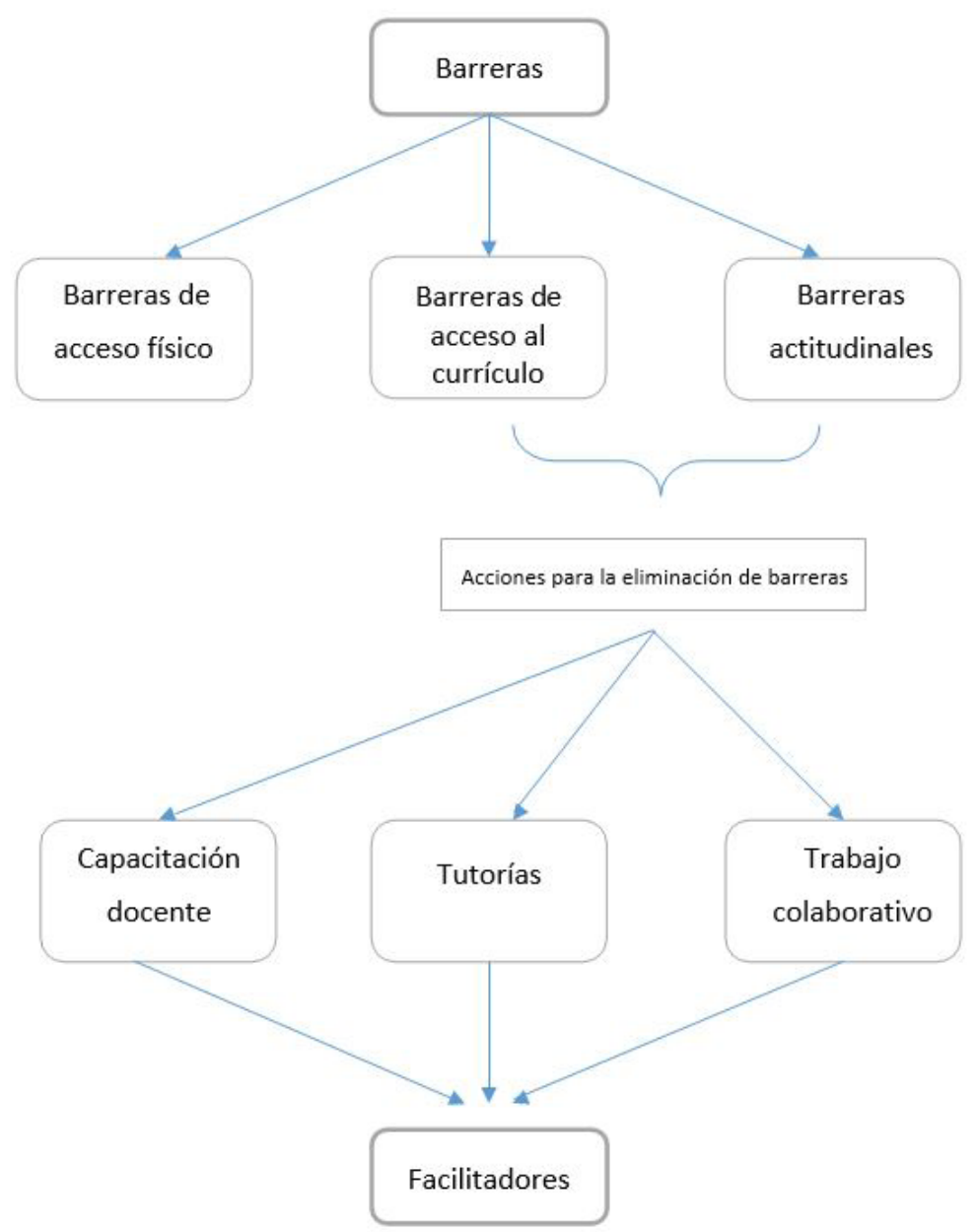

Figura $N^{\circ} 3$

Tercer nivel de análisis

\section{Resultados}

En los análisis de primer, segundo y tercer nivel se utilizaron citas para corroborar y fortalecer la investigación.

3.1. Primer nivel de análisis: construcción de categorías

3.1.1. Categoría: Proceso de admisión 
3.1.1.1. Programas de transición entre educación media y educación superior

Se puede inferir del discurso de los representantes de la RESI que, en general, no se han establecido programas para acompañar, guiar o ayudar a estudiantes en situación de discapacidad (EsD) en su proceso de transición y posterior incorporación a la educación superior. Sin embargo, se observa en el discurso la intención y necesidad de proponer y crear programas que tengan como objetivo el acompañamiento desde la enseñanza media para facilitar el ingreso y permanencia de los estudiantes en la educación superior.

No, nuestra universidad no posee un programa de transición, pero recientemente hemos tenido reuniones con establecimientos para coordinarnos y comenzar a establecer las primeras propuestas para apoyar a los EsD que desean ingresar a la educación superior. (Entrevista $N^{\circ}$ 6, 1)

No existe un programa propiamente tal, pero el Instituto realiza talleres en liceos para facilitar la admisión de estudiantes con o sin discapacidad. (Entrevista No 9, 1)

No obstante, cuatro instituciones de educación superior hicieron mención del Programa de Acompañamiento y Acceso Efectivo a la Educación Superior (PACE), como un programa de transición que busca asegurar el acceso a la educación. Dentro de este contexto se enfatiza que el programa PACE tiene entre sus requisitos las notas de enseñanza media, la asistencia, la ubicación geográfica del establecimiento educacional, entre otros, pero la situación de discapacidad no asegura ni garantiza cupos en las carreras y programas de la oferta académica.

La universidad no tiene ingreso especial. Todos los estudiantes deben ingresar vía PSU. No obstante, pueden ingresar por PACE, deben rendir la PSU, pero el puntaje no es considerado, se les asegura el ingreso a la educación superior por sus buenas notas, a veces coincide que los estudiantes PACE tienen discapacidad. (Entrevista $N^{\circ}$ 6, 4) 
Igualmente, una de las instituciones participantes de este estudio posee un programa de transición entre enseñanza media y educación superior denominado "Propedéutico", destinado a estudiantes sordos, y consiste en realizar un año de nivelación para su posterior ingreso a la educación superior.

Respecto de las personas sordas que se comunican a través de lengua de señas, no hay un referente como tal en Chile, por lo tanto, lo que nosotros generamos como programa es un proceso de transición, que nosotros denominamos "Propedéutico: Programa de Lengua de señas y español", que tiene que ver con la alfabetización del español y el incremento técnico de la lengua de señas chilena, cuando se puede ambas, o nos inclinamos por una cuando el estudiante siempre trabajó con lengua de señas. (Entrevista $N^{\circ} 1,1$ )

\subsubsection{Admisión especial}

Para dar cumplimiento al derecho a la educación, las instituciones de educación superior aquí representadas han establecido medidas para el ingreso de personas en situación de discapacidad a través de sistemas de admisión especial. Si bien algunas instituciones también incorporan a deportistas de alto rendimiento, extranjeros o estudiantes egresados de otras carreras, resulta ser la medida más utilizada en casos de discapacidad sensorial, ya que los casos de discapacidad física generalmente ingresan vía PSU adaptada o a través de vías de admisión tradicional.

Por otra parte, también existen instituciones que matriculan a los estudiantes sin contemplar los resultados obtenidos en la PSU e incluso sin la necesidad de haberla rendido, delegando la decisión de ingreso a un grupo de personas encargadas de admisión en el que también participan profesionales de los equipos de inclusión.

No hay un sistema de ingreso especial para personas en situación de discapacidad. Existe un sistema de ingreso especial para extranjeros, deportistas, intercambios dentro de carreras y personas en situación de discapacidad. Además, cualquier persona con discapacidad puede ingresar a la unidad estudiantil, a través de PSU (...). (Entrevista $\left.\mathrm{N}^{\mathrm{o}} 8,4\right)$ 
La Institución 2 posee un programa de admisión especial para personas con discapacidad visual, creado el año 2001, siendo el único programa de admisión especial específico para una discapacidad.

La universidad cuenta con el ingreso especial para personas con discapacidad visual. A través de la Unidad de Admisión los estudiantes pueden postular sin necesidad de PSU. El año 2001 se crea el decreto de admisión especial para personas con discapacidad visual, por eso es tan específico. (Entrevista No 3, 2)

En aquellas instituciones de educación superior en las que el ingreso no se realiza mediante PSU o poseen matrícula a distancia, es ampliamente utilizado el llenado de una ficha al momento de la matrícula, para entregar información respecto de la situación de discapacidad y si el EsD posee registro nacional de discapacidad (RND).

Al matricularse, online o presencial, los estudiantes deben responder tres preguntas relacionadas con discapacidad, si poseen credencial o si participaron del PIE en su colegio. Si alguna de estas preguntas es positiva, los estudiantes tienen la posibilidad de acceder a la unidad de inclusión y ser evaluados para saber si son candidatos para recibir los apoyos. Se les invita a ser parte de la unidad. (Entrevista No 8, 18)

\subsubsection{Requisitos para la postulación}

Los requisitos para la postulación difieren significativamente entre una institución y otra. Contar con credencial, estar inscrito en el RND y la licencia de enseñanza media son el factor común entre los requerimientos; además, deben asistir a una entrevista personal con las encargadas de inclusión, las jefaturas de carrera y/o la dirección de escuela. En esta entrevista se extrae información importante respecto de competencias y necesidades de postulantes; asimismo, es posible determinar qué tipos de adecuaciones arquitectónicas o de acceso se deben realizar en la institución en caso de ingreso del postulante.

Los estudiantes postulan, se realiza un encuentro informativo, una entrevista con la encargada de inclusión para conocer aspectos vocacionales, información de la carrera, las proyecciones. Se les informa con lo que cuenta la universidad, la encargada de inclusión 
realiza un informe verbal o por correo y se lo entrega a los directores de carrera. Los estudiantes se entrevistan con los directores de carrera y/o programa. Luego se entregan los resultados de postulación y finalmente se procede a matricular y orientar. (Entrevista $\mathrm{N}^{\circ} 4,4$ )

Es posible deducir que la mayoría de las instituciones no solicita la documentación de los Programas de Integración Escolar (PIE); asimismo, no incorpora entre los requisitos para postular los informes, evaluaciones, protocolos e historial en el PIE.

La evidencia acumulada permite reafirmar que los programas de admisión para personas en situación de discapacidad de las instituciones de educación superior son heterogéneos, ya que los criterios utilizados para evaluar el ingreso varían en relación con la carrera que entrega el acceso y algunas instituciones ponen restricciones al tipo de discapacidad presentada por el solicitante.

3.1.1.4. Disponibilidad y accesibilidad de información acerca de programas de admisión e ingreso de personas en situación de discapacidad a la educación superior

La totalidad de los participantes expone que en las páginas web institucionales existe información relativa a los programas especiales de admisión, programas de inclusión y programas de apoyo dependientes de otros departamentos. Las redes sociales son también una estrategia utilizada para entregar a la comunidad información de las acciones y programas de inclusión.

Sí hay, la página tiene una plataforma con los ingresos especiales, los requisitos y los plazos. La página no es cien por ciento accesible. (Entrevista No 3, 3)

Sí, a través de la página web. Si bien, no es tan accesible, sin embargo, a través de un software se puede acceder a ella. (Entrevista $N^{\circ} 8,3$ )

La importancia de difundir —a través de redes sociales, páginas web, afiches y/o stands- la existencia de equipos de apoyo a la inclusión y de ser reconocidos por la comunidad educativa y por personas con discapacidad está relacionado íntimamente con 
promover el acceso a la información en igualdad de oportunidades y la autonomía. No obstante, no existe información accesible en páginas web institucionales que describa los procesos de admisión, requisitos y fechas de postulación a instituciones de educación superior, utilizando macrotextos, lengua de señas u otros idiomas, contraviniendo criterios de accesibilidad de la World Wide Web Consortium para desarrollar estándares web internacionales.

3.1.2. Categoría: programas de apoyo a estudiantes en situación de discapacidad

\subsubsection{Programa de inclusión}

La reciente y creciente conformación de equipos de inclusión en instituciones de educación superior da cuenta de la relevancia que ha cobrado la premisa de igualdad de oportunidades en términos de inclusión educativa. La tabla $N^{o} 1$ muestra que en la región del Biobío existen programas e iniciativas destinados a servir de guías y dar apoyo al alumnado con discapacidad. Estos programas se han conformado a través de voluntariados y como consecuencia del ingreso de EsD. Por su parte, existen también instituciones que, sin contar con un programa de inclusión, realizan acciones en favor de los EsD, generalmente articuladas con otros programas de apoyo estudiantil y que consideran que la discapacidad es parte de la gran diversidad existente entre sus estudiantes.

\section{Tabla $\mathrm{N}^{\circ} 1$.}

Programas o departamentos de inclusión de las instituciones de educación superior pertenecientes a la RESI

\begin{tabular}{ll}
\hline Institución & Programa de inclusión o departamento afín \\
\hline Institución 1 & $\begin{array}{l}\text { Programa general de inclusión } \\
\text { Institución 2 }\end{array}$ \\
$\begin{array}{l}\text { Programa general de inclusión } \\
\text { Institución 3 }\end{array}$ & $\begin{array}{l}\text { Dirección de Educación Inclusiva } \\
\text { Coordinación Nacional de Inclusión } \\
\text { Institución 4 }\end{array}$ \\
/Comité de inclusión en cada sede \\
Institución 5 & Programa de inclusión \\
Institución 6 & $\begin{array}{l}\text { Incluido en otro programa de apoyo estudiantil } \\
\text { Institución 7 }\end{array}$ \\
Institución 8 & Sin programa de inclusión estudiantil \\
\hline
\end{tabular}

Fuente: Elaboración propia, 2019. 


\subsubsection{Recursos económicos}

Los recursos económicos destinados a los programas de inclusión se obtienen de distintos fondos. Lo más común es el financiamiento interno, destinado al pago de honorarios y mantención del espacio físico y material de oficina. También existen programas financiados con proyectos internos, los que deben ser repostulados al término del periodo. En algunos casos, los encargados de inclusión forman parte de otros programas, por lo que su cargo no es exclusivo.

No hay recursos destinados específicamente a este item, pero hay recursos para todos los estudiantes según sus necesidades, para favorecer su participación (...). (Entrevista No 5,14 )

En cuanto a los recursos designados para ayudas técnicas y material de apoyo, la totalidad de las instituciones postulan a fondos externos, generalmente financiados por el Servicio Nacional de Discapacidad (SENADIS). Estos fondos son concursables y abren postulaciones todos los años. Los representantes de las instituciones de educación superior comentan que, mediante los proyectos de SENADIS, financian principalmente ayudas técnicas de alto costo, capacitaciones docentes o adecuaciones arquitectónicas para mejorar la accesibilidad.

Respecto de la capacidad para cumplir con los requerimientos de los estudiantes que lo demanden, contando con la cantidad de ayudas técnicas suficientes para todos, la mayoría de los representantes indican que no ha sido necesario y por dos razones principales: que la adquisición de material y ayudas técnicas se realiza en la medida que se requiere y según la contingencia, y que se insta a los estudiantes a postular a proyectos de SENADIS de manera individual, con el objetivo de que las ayudas técnicas adjudicadas pertenezcan a ellos y puedan ser utilizadas en cualquier contexto.

No, porque no es necesario, nosotros siempre orientamos a los estudiantes para que postulen a SENADIS y tener sus propios instrumentos, sus propias ayudas técnicas, porque no solo les van a servir acá, si no también en su vida diaria. Pero cuando no tienen y lo necesitan, nosotros se lo prestamos por el año o por un semestre. La idea 
es que siempre ellos vayan adquiriendo sus ayudas técnicas, por lo tanto, no sería necesario tener una por estudiante. (Entrevista $N^{\circ} 3,11$ )

\subsubsection{Recursos humanos}

Los equipos de inclusión, en su génesis, fueron en su gran mayoría conformados por voluntarios o personal de las instituciones de educación superior que ocupaban otros cargos íntimamente relacionados con estudiantes. Por lo general, pertenecían a bienestar estudiantil o a departamentos de apoyo al estudiante. Con el tiempo y al reconocer la necesidad de contar con horas exclusivas para el apoyo a la diversidad, se han ido conformando programas que cuentan con un profesional a cargo o equipos conformados hasta por 14 profesionales.

La tabla $\mathrm{N}^{\circ} 2$ muestra los profesionales que componen los equipos de inclusión o bien los encargados de dar apoyo a los EsD matriculados en las instituciones que participaron de esta investigación, siendo el educador diferencial el profesional con más presencia en las instituciones.

Tabla No 2.

Profesionales que componen el equipo de inclusión en las instituciones de educación superior pertenecientes a la RESI

\begin{tabular}{|c|c|c|c|c|c|c|c|c|}
\hline & 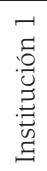 & 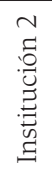 & 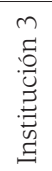 & 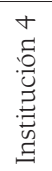 & 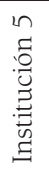 & 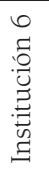 & 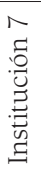 & 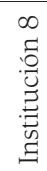 \\
\hline Educador diferencial & $\mathrm{X}$ & $\mathrm{X}$ & $\mathrm{X}$ & $\mathrm{X}$ & $\mathrm{X}$ & $\mathrm{X}$ & $X$ & \\
\hline Psicólogo & $\mathrm{X}$ & & & $\mathrm{X}$ & & & $\mathrm{X}$ & \\
\hline Terapeuta ocupacional & & & & $\mathrm{X}$ & & & & \\
\hline Kinesiólogo & $\mathrm{X}$ & $\mathrm{X}$ & & & & & & \\
\hline Fonoaudiólogo & $\mathrm{X}$ & & & & & & & \\
\hline $\begin{array}{l}\text { Trabajador social/ } \\
\text { Técnico trabajo social }\end{array}$ & $\mathrm{X}$ & $\mathrm{X}$ & & & & & & $\mathrm{X}$ \\
\hline Psicopedagogos & & & & $\mathrm{X}$ & & & & \\
\hline Profesor general & & & & $\mathrm{X}$ & & & & \\
\hline Diseñador gráfico & & & & $\mathrm{X}$ & & & & \\
\hline Intérprete lengua de señas & $\mathrm{X}$ & & & & & & & \\
\hline
\end{tabular}

Fuente: Elaboración propia, 2019. 


\subsubsection{Categoría: Accesibilidad}

\subsubsection{Accesibilidad para el acceso físico}

Los representantes de la RESI, en su mayoría, indican que se han realizado ajustes físicos de acceso en la medida que se requieren. Esto tiene relación con los altos costos que implica instalar ascensores, rampas o rutas accesibles entre un edificio y otro, sobre todo en aquellas instituciones en las que sus edificios son antiguos o declarados patrimonio nacional.

Tenemos edificios accesibles, pero no todos. En los antiguos aún se están realizando ajustes razonables. Todos los edificios nuevos están diseñados y construidos según el decreto 50. Aún falta por hacer. (Entrevista $\mathrm{N}^{\circ} 1,12$ )

Se intenta, pero no. Aún existen accesos que unen edificios con inclinaciones que no permiten el desplazamiento autónomo. Estacionamiento no cumple con las medidas y tiene piedrecillas. El acceso a biblioteca es casi plano, pero un escalón de $2 \mathrm{~cm}$ no permite a una silla de ruedas subir. Y la rampa está hecha para una sola puerta. (Entrevista $\mathrm{N}^{\circ} 7,12$ )

En general, en cuanto a la accesibilidad a espacios físicos, es necesario contar con una visión global respecto de la construcción, adecuación y desarrollo de espacios, ya que aún faltan ajustes por hacer, por ejemplo, contar con al menos un baño accesible por edificio y acceso a bibliotecas, casinos, auditorios, laboratorios y espacios comunes, con la finalidad de que todas las personas, sin distinción, puedan hacer uso de ellos.

\subsubsection{Accesibilidad para acceder al currículo}

Dependiendo de los lineamientos en que se enmarque el equipo de inclusión, las adecuaciones curriculares no siempre se encuentran entre las estrategias más utilizadas para apoyar al EsD. Esto depende del enfoque que posea el equipo de inclusión y la disponibilidad del docente para realizar adecuaciones. Existen equipos que no trabajan directamente con docentes y se limitan a la realización de charlas, talleres o capacitaciones con temáticas de accesibilidad al currículo; 
no obstante, no realizan seguimiento a las prácticas inclusivas ni pueden exigir el cumplimiento de las estrategias propuestas.

Es lo que se pretende, pero no se puede asegurar que se haga. Las capacitaciones también se realizan entregando las herramientas para que se preparen las clases pensando en la diversidad. (Entrevista $\mathrm{N}^{\circ}$ $2,25)$

Los docentes realizan sus clases como ellos lo consideran pertinente, por lo tanto, lo importante es cómo acceden a la información. (Entrevista $\left.\mathrm{N}^{\circ} 3,25\right)$

Esto puede deberse al desconocimiento del docente, ya que existen prácticas pedagógicas que no se adecuan a las necesidades de los estudiantes y a su derecho a una evaluación diferenciada. Los datos obtenidos en las entrevistas indican que no siempre se llevan a cabo las adecuaciones o, bien, se realizan cuando la necesidad ya está instaurada, por lo que los EsD son evaluados de la misma manera que sus compañeros.

Por lo general, no. Porque para los docentes sus estudiantes son todos iguales, no hay diferencia, entonces cuando ven que las diferencias se están notando, ahí ellos recién deciden ayudar o cooperar. No sé si se podrá ayudar más, pero los docentes cuando ven necesidades siempre lo hacen. (Entrevista No 9, 25)

Los decretos 170 y 83 del Ministerio de Educación entregan las orientaciones para el trabajo con estudiantes con necesidades educativas especiales, su derecho a acceder al currículo y a una evaluación diferenciada. Empero, en la educación superior no es posible desarrollar una adecuación más profunda del currículo, considerando que para optar a una carrera técnica o profesional no pueden quedar contenidos sin abordar. Por lo tanto, la opción es la adecuación de acceso al currículo, la que queda sujeta a la disponibilidad y voluntad de los docentes.

Eso no te lo podría asegurar, porque depende de cada docente. Se propicia de que sea así, no está declarado el DUA porque la malla 
está basada pensando en el desarrollo de competencias. (Entrevista $\left.\mathrm{N}^{\circ} 5,25\right)$

Por otra parte, el acceso al currículo de personas con discapacidad visual o auditiva se caracteriza principalmente por adecuaciones de acceso a la información, tanto de material de apoyo como a través de ayudas técnicas e intérprete de lengua de señas en la sala de clases.

En el caso de personas con baja visión, la totalidad de las instituciones de educación superior que han contado o cuentan con EsD visual, poseen las ayudas técnicas necesarias y suficientes para dar respuesta a las necesidades de acceso a la información. Además, apoyan a sus estudiantes en postulación a proyectos SENADIS para adquirir sus propios materiales según su grado de discapacidad visual.

Asimismo, cinco de las seis instituciones de educación superior que cuentan con EsD auditiva han contratado intérprete de lengua de señas y apoyado a los estudiantes en la postulación a proyectos SENADIS para contar con su propio intérprete, financiado a través de este medio. Igualmente, existe una institución de educación superior que cuenta con EsD auditiva, que tiene entre sus profesionales cuatro intérpretes de lengua de señas, un coordinador técnico de intérprete y un instructor de lengua de señas.

Se contrata en función de la necesidad. En estos momentos no hay porque no se requiere, pero en Santiago sí existen y la universidad lo gestiona y lo financia. Idealmente, el estudiante trae a su intérprete por temas de comodidad, vinculo y comunicación. Se procura que lo hagan a través de SENADIS, que sea alguien de su agrado, si eso no ocurre, se contrata desde la universidad. (Entrevista $\mathrm{N}^{\circ} 7,9$ )

Es importante consignar que no todas las discapacidades auditivas requieren de un intérprete de lengua de señas y que, dependiendo del grado de discapacidad, pueden requerir solo ayudas técnicas, como amplificadores de sonido, audífonos y/o micrófono. 
3.2. Segundo nivel de análisis: eficiencia

3.2.1. Matrícula de estudiantes en situación de discapacidad, año 2019

Tabla $N^{0} 3$.

Matrícula de estudiantes en situación de discapacidad al año 2019

\begin{tabular}{lc}
\hline Institución de educación superior & $N^{o}$ matriculados 2019 \\
\hline Institución 1 & 73 \\
Institución 2 & 187 \\
Institución 3 & 22 \\
Institución 4 & 9 \\
Institución 5 & 7 \\
Institución 6 & 36 \\
Institución 7 & 82 \\
Institución 8 & 9 \\
\hline
\end{tabular}

Fuente: Elaboración propia, 2019.

\subsubsection{Diagnósticos}

De acuerdo con la tabla $\mathrm{N}^{0} 4$, la discapacidad física o motora sigue teniendo la mayor cantidad de matriculados en la educación superior. Por su parte, la discapacidad psíquica, que incluye a los estudiantes diagnosticados con Trastorno del Espectro Autista (TEA), ha tenido un crecimiento exponencial. Le siguen la discapacidad visual, auditiva (sensorial) y, por último, la discapacidad intelectual y discapacidad múltiple. 
Tabla $\mathrm{N}^{0} 4$.

Número de estudiantes matriculados en instituciones de educación superior al año 2019 clasificados por discapacidad

\begin{tabular}{|c|c|c|c|c|c|c|c|c|c|}
\hline & 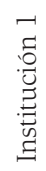 & 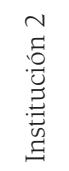 & 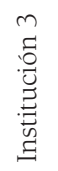 & 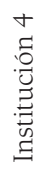 & 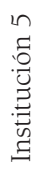 & 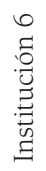 & 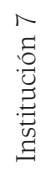 & 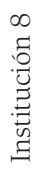 & TOTAL \\
\hline $\begin{array}{l}\text { Discapacidad } \\
\text { visual }\end{array}$ & 1 & $80 *$ & \multirow[b]{2}{*}{11} & 3 & --- & 3 & 8 & --- & \multirow[b]{2}{*}{166} \\
\hline $\begin{array}{l}\text { Discapacidad } \\
\text { auditiva }\end{array}$ & 28 & 18 & & 1 & 1 & 1 & 11 & --- & \\
\hline $\begin{array}{l}\text { Discapacidad } \\
\text { motora }\end{array}$ & 22 & 50 & 9 & 5 & --- & 3 & 40 & 2 & 131 \\
\hline $\begin{array}{l}\text { Discapacidad } \\
\text { psíquica/TEA }\end{array}$ & 1 & 35 & 2 & --- & 5 & 29 & 16 & 1 & 89 \\
\hline $\begin{array}{l}\text { Discapacidad } \\
\text { múltiple }\end{array}$ & 1 & --- & --- & --- & --- & --- & --- & --- & 1 \\
\hline $\begin{array}{l}\text { Discapacidad } \\
\text { intelectual }\end{array}$ & 20 & --- & --- & --- & --- & --- & 2 & 6 & 28 \\
\hline $\begin{array}{l}\text { Discapacidad } \\
\text { sin definir }\end{array}$ & --- & 4 & --- & --- & 1 & --- & 5 & --- & 10 \\
\hline *21 estudiant & nd & por 1 & gram & xclu & 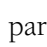 & sca & $\mathrm{ad}$ & & 425 \\
\hline
\end{tabular}

Fuente: Elaboración propia, 2019.

Estos datos corresponden a cifras entregadas por los representantes de la RESI. Sin embargo, en su discurso fueron enfáticos al declarar que no todos los EsD se encuentran detectados o forman parte de los equipos de inclusión, ya que su participación es voluntaria y muchos de ellos optan por no recibir apoyo, por lo que este número podría ser mayor.

\subsubsection{Registros históricos}

Las instituciones de educación superior, en su mayoría, no cuentan con registros históricos de ingresos, egresos o deserción de EsD. Si bien las instituciones poseen registros de todos sus estudiantes, no se han precisado cifras que hablen específicamente de EsD. 
Estamos trabajando en un registro. No tenemos cifras específicas. Tenemos datos muy generales. Tenemos un registro desde el año 2015. (Entrevista No 3, 29)

Al interior de los discursos se deja entrever la importancia de contar con registros históricos, ya que estas cifras dan cuenta de la creciente matrícula y de la necesidad de poner en marcha nuevas estrategias de trabajo en apoyo a la diversidad, además de contar con más recursos que mejoren las condiciones de estudiantes, docentes y equipo de apoyo a la inclusión.

Sí, existen registros, pero de todos los estudiantes, no específicamente de los EsD, sería bueno contar con esas cifras. (Entrevista $N^{0} 5,29$ )

\subsection{Tercer nivel de análisis: barreras y facilitadores}

\subsubsection{Barreras}

\subsubsection{Barreras de acceso físico}

Las barreras de acceso físico son siempre las más visibles y las que se detectan con mayor facilidad. Aunque parecen ser fáciles de corregir, por una parte se requieren altas sumas de dinero para realizar ajustes razonables y, por otra, ciertas instituciones de educación superior poseen edificios patrimoniales, que no pueden ser modificados sin realizar diligencias que consideren el nivel de accesibilidad existente y el requerido para maximizar la preservación de las características y así mejorar las condiciones de accesibilidad.

En general, las exigencias relacionadas con acceso físico a edificios principales, tales como bibliotecas, salas de clases y casinos, cumplen con la normativa y han sido satisfechas mediante la instalación de rampas o rutas accesibles. No obstante, en todas las instituciones que incorporan estudiantes a carreras científicas falta realizar ajustes razonables al mobiliario, en espacios utilizados para laboratorios o talleres técnicos, con accesibilidad en las salidas a terreno o en aquellos lugares en los que se realizan prácticas profesionales. 
Asimismo, aún existen instituciones que no cumplen con las medidas básicas de accesibilidad, ya sea por la geografía en la que están inmersos los edificios, porque aún no cuentan con estudiantes que requieran acceder a sus instalaciones o porque realizan ajustes sin la necesidad de intervenir la arquitectura, por ejemplo, trasladando a los EsD a los primeros pisos de los edificios.

Otro de los elementos de interferencia es la falta de una adecuada guía de inducción al incorporarse a la institución. Idealmente, un EsD requiere de una figura que acompañe y muestre las rutas accesibles a los diferentes recintos que necesite utilizar. Sin embargo, esta práctica no está instaurada en todos los planteles, ya que en algunos se realiza de manera voluntaria, a veces por miembros del equipo de inclusión, por docentes o por los propios compañeros.

\subsubsection{Barreras de acceso al currículo}

Las barreras de acceso al currículo están mediadas, esencialmente, por la falta de estrategias metodológicas y, por lo tanto, dependen en gran medida del conocimiento y la experiencia del docente en adecuaciones de acceso, inclusión, DUA y tipos de adaptaciones factibles de realizar según discapacidad, así como también de prácticas instaladas que tengan las instituciones para apoyar la labor docente y capacitaciones que impartan.

Respecto del uso de tecnología en la sala de clases para apoyar el acceso al currículo, su utilización está supeditada a la incorporación de los EsD y la consecuente necesidad de aprender a utilizar tecnología acorde con las características del estudiante. Además, la falta de instancias de capacitaciones y talleres relacionados con esta temática propende a homogeneizar la enseñanza y a condicionar el aprendizaje según las estrategias y modalidades propias de cada docente.

\subsubsection{Barreras actitudinales}

Las expectativas que los docentes tienen de los EsD están mediadas por creencias que tienen de personas en situación de discapacidad; además, ello tiene relación con las oportunidades de aprendizaje que 
brinda el docente al estudiante. Por eso, las barreras actitudinales cobran un rol protagónico a la hora de aplacar aquellos prejuicios aún presentes en la comunidad educativa.

... aún existen algunos docentes con una visión de estudiante-profesor o de educación totalmente fuera de la inclusión, por lo tanto, hay lenguaje poco inclusivo, visión de docentes muy lejos de la inclusión, que consideran que algunos estudiantes no debieran ser aceptados en la universidad. (Entrevista $N^{\circ} 8,16$ )

Lo anterior tiene estrecha correspondencia con la necesidad del cambio de paradigma que se desea instaurar respecto de la incorporación de EsD a la educación superior, lo que se ve acentuado, según los entrevistados, con la falta de políticas inclusivas que fomenten la participación de personas con discapacidad, y que incluyan no solo a académicos, sino también a administrativos y estudiantes en capacitaciones, talleres, charlas y seminarios que ayuden a visibilizar la diversidad.

Y, por otra parte, el desconocimiento de nuestro equipo académico, que muchas veces no nos preparan para trabajar con la diversidad y, por lo tanto, nos preparan para un perfil de estudiantes caracterizado con ciertos lineamientos que son la generalidad de un estudiante de educación superior, pero que no considera esta diversidad. ¿Cómo yo, como académico, apoyo a una persona ciega que entra a mi carrera? ¿Y cómo yo visualizo, en términos de egreso y empleabilidad, qué va a ser esa persona, no generando los prejuicios asociados a algunas discapacidades? Creo que tiene que ver con desconocimiento de cómo poder generar adecuaciones metodológicas y por desconocimiento de la proyección laboral que derribe ciertos prejuicios. (Entrevista $\mathrm{N}^{\circ} 5,16$ )

Por consiguiente, un componente que obstruye el trabajo con la diversidad son las prácticas académicas que tienden a la homogenización. El estudio permite inferir que algunos docentes, en su mayoría con más años de experiencia, presentan un proceso pedagógico homogeneizante, desarrollándose estilos de enseñanzas centrados en habilidades y destrezas, desconociendo el carácter diverso, variado y diferente de todos los estudiantes, y por ende el contexto educativo. 
Existen barreras actitudinales, con prácticas pedagógicas muy antiguas y rígidas. Aqui hay docentes muy añosos. (Entrevista No 6, 16)

Existe una barrera actitudinal que concierne a los docentes y que se refiere al interés por participar de las capacitaciones relacionadas con inclusión y diversidad. Las instituciones realizan capacitaciones optativas que tributan a docencia y la invitación es abierta a toda la comunidad académica. No obstante, la asistencia no es la esperada, ya que no todos sienten la necesidad de asistir y perfeccionarse, hasta que se encuentran con un EsD en sus aulas y solo en ese momento se acercan a solicitar apoyo a los equipos de inclusión. Según el discurso expuesto en las entrevistas, son siempre los mismos docentes que participan de las capacitaciones.

\subsubsection{Facilitadores}

\subsubsection{Capacitación docente}

Las instituciones de educación superior cuentan, en su mayoría, con departamentos cuya función es capacitar a los académicos en diversas áreas relacionadas con su disciplina y carrera. No obstante, la falta de especificidad en temas relacionados con inclusión, metodologías y estrategias inclusivas ha hecho que sean los equipos de inclusión los que tomen la iniciativa y decidan incorporar entre sus funciones la realización de talleres y charlas para concientizar y apoyar la diversidad.

En el discurso de los representantes de la RESI se hace explícita la necesidad de capacitación constante para trabajar con los EsD, pues se trata no solo de ellos y su perseverancia para cumplir con los objetivos propuestos. La realidad es que se requieren estrategias que los docentes reconocen no tener y que son susceptibles de desarrollar. La necesidad de capacitación es evidente, no solo por estar explícita en todos los discursos, sino también porque, en la práctica, tratar problemas específicos no es parte de las competencias de los docentes, lo que significa que las estrategias o adecuaciones curriculares muchas veces no son las más eficaces o no se realizan. Frente a estas necesidades, algunas instituciones han puesto a disposición de los docentes, diplomados gratuitos que incorporan temáticas de inclusión. 


\subsubsection{Tutorías y mentorías}

Son estrategias utilizadas en la mayoría de las instituciones para apoyar estudiantes que requieren de un soporte adicional para lograr adquirir las habilidades conceptuales, procedimentales y actitudinales. La principal característica de la mentoría es que la realizan sus pares, estudiantes de la misma carrera o afines, de semestres más avanzados, que apoyan el aprendizaje. Asimismo, refieren la modalidad de tutorías realizadas por profesionales, generalmente recién egresados, en especial en carreras científico-matemáticas o de ingeniería, que requieren mayor manejo de contenidos.

Igualmente, se destaca el acompañamiento que realizan los mentores a estudiantes con Trastorno del Espectro Autista, generalmente de alto funcionamiento que, si bien poseen las habilidades académicas conceptuales y procedimentales, fallan enormemente en las habilidades sociales; por consiguiente, deben ser reforzadas las competencias comunicativas, de sociabilización, y las estrategias para establecer relaciones interpersonales, las que pueden ser potenciadas por los tutores pares, previa inducción desde los equipos de inclusión.

\subsubsection{Trabajo colaborativo}

Frente a las exigencias que supone trabajar con estudiantes tan diversos, resulta fundamental el trabajo en equipo. Para lograrlo se necesitan instancias que promuevan el intercambio de experiencias y estrategias, y una de las modalidades es a través del trabajo colaborativo, para lo cual se requiere de instituciones que promuevan y brinden espacios y tiempos necesarios para llevarlo a cabo. Asimismo, se requiere de un equipo de gestión que escuche las necesidades de los docentes, recoja sus inquietudes y solicitudes, y trabaje para construir una institución generadora de soluciones y abierta al diálogo.

\section{Discusión}

Esta investigación tuvo como objetivo conocer cómo operan las instituciones educativas en los procesos de inclusión de EsD, 
cómo acceden estos alumnos al currículo y las barreras de acceso al aprendizaje y a la participación que encuentran al momento de su incorporación. Para ello se analizó el discurso de los representantes de RESI acerca de las acciones que se llevan a cabo en instituciones de educación superior en la región del Biobío.

Sobre los programas de admisión para EsD, podemos concluir que no poseen un sistema de admisión especial unificado, ya que los criterios utilizados para evaluar el ingreso varían en relación con la carrera a la que entregan el acceso y algunas instituciones ponen restricciones al tipo de discapacidad presentada por el solicitante. Estos hallazgos refrendan lo descrito por la literatura respecto de las dificultades en el proceso de admisión a la educación superior, el cual no se encuentra unificado ni cuenta con las garantías mínimas de accesibilidad, siendo una de las falencias en cuanto a inclusión educativa (Lizama, Gil y Rahmer, 2018).

Con relación a la accesibilidad, si bien la mayoría de las instituciones posee adecuaciones de acceso físico básicas para recibir EsD, estas se han ido realizando en la medida que son requeridas, es decir, cuando el EsD se encuentra ya matriculado, lo que ha provocado que no todos los recintos sean accesibles y que existan barreras de acceso físico, especialmente en laboratorios, auditorios y espacios técnicos que aún no cuentan con estudiantes o que recientemente han ido incorporando (Victoriano, 2017).

En un nivel más particular, respecto del acceso al currículo desde las ayudas técnicas, la totalidad de las instituciones de educación superior cuenta con la cantidad suficiente de material para EsD. Destaca el apoyo brindado por SENADIS, al entregar fondos que las instituciones se adjudican a través de proyectos, los que van en directo beneficio de los estudiantes, permitiendo acceder a material técnico de propiedad de las instituciones y favoreciendo la postulación a ayudas técnicas que quedarán en posesión de los estudiantes.

En cuanto a las adecuaciones al currículo, los decretos 170/2010 y 83/2015 rigen en los niveles parvulario, básico y medio, 
dejando un vacío en la enseñanza superior, porque no se puede adecuar los resultados de aprendizaje, lo que lleva a realizar ajustes razonables o, en su defecto, adecuaciones curriculares de acceso.

Respecto de la categoría de eficiencia, referida a los diagnósticos de EsD, se extrae que el ingreso estaba restringido a personas con discapacidad física, que requerían adecuaciones de acceso físico más que curricular. Asimismo, la postulación estaba condicionada a ciertas carreras, generalmente humanistas y sociales. Pero esto ha ido cambiando, lo que se ve reflejado tanto en la literatura como en este estudio, en que la incorporación de EsD sensorial, psíquica e intelectual es cada vez más habitual (Paz-Maldonado, 2020).

En relación con las barreras, algunas investigaciones que exponen el actual escenario de la inclusión y atención a la diversidad en educación superior no son alentadoras. Por una parte, los EsD en instituciones de educación superior son escasos y quienes ingresan se enfrentan a limitaciones arquitectónicas, pedagógicas, organizativas, conductuales y/o actitudinales. La inflexibilidad de los académicos, la falta de capacitación al personal y la poca accesibilidad son las principales barreras (Castro et al., 2016). A pesar de las leyes actuales, existe un vacío caracterizado por la ausencia de una normativa y políticas inclusivas al interior de los planteles.

Sumado a esto, debemos considerar que, sin una política interna que entregue los lineamientos básicos para la planificación de clases y la elaboración de evaluaciones para dar respuesta a la diversidad, parece imposible exigir a los docentes que cumplan con los estándares mínimos de accesibilidad al currículo, lo que lleva a reflexionar acerca del rol docente y su responsabilidad para adecuarse a la diversidad. Esta falta de políticas internas impide asegurar el acceso universal al currículo y, por el contrario, relega la responsabilidad en cada docente, de acuerdo con su voluntad, interés y compromiso con los EsD. En definitiva, no todas las instituciones de educación superior que cuentan con EsD elaboran estrategias para el acceso al currículo ni disponen de materiales curriculares apropiadamente adaptados para los estudiantes (Fajardo, 2017). Sin embargo, se reportan algunas experiencias excepcionales, de casos 
"exitosos", que cuentan con profesores que escuchan, se interesan y se acercan a sus estudiantes.

Otro aspecto que merece ser discutido es la ausencia de prácticas reflexivas que impiden fortalecer el trabajo colaborativo; aun existiendo las instancias en la mayoría de las instituciones, estas no dedican el tiempo suficiente a temas de inclusión, centrándose en mejorar las estrategias y metodologías de sus clases, sin contemplar a los EsD (Victoriano, 2017).

Por último, en relación con las barreras, los programas de inclusión responden a las necesidades de los estudiantes desde las ayudas técnicas y también desde el apoyo directo; sin embargo, en su mayoría, el trabajo con el docente se limita a capacitaciones, charlas y talleres. La baja participación de los docentes en las capacitaciones ha impedido contar con docentes involucrados con la diversidad y, en ocasiones, hasta se han convertido en la barrera más compleja de derribar, ya que los prejuicios y las bajas expectativas de los docentes entorpecen los procesos con los EsD (Salinas, Lissi, Medrano, Zuzulich y Hojas, 2013).

A partir del análisis de los facilitadores, los resultados sugieren que existen diferencias entre una institución y otra. Mientras algunas han fortalecido los equipos de inclusión, incorporando profesionales de apoyo expertos en la materia, otras han destinado sus recursos al perfeccionamiento y capacitación docente, y otras tantas han financiado ayudas técnicas o realizado ajustes razonables a sus edificios para mejorar la accesibilidad.

Uno de los facilitadores más utilizados son las tutorías entre pares, las que destacan por su bajo costo o modalidad a través de voluntariado; además, no necesitan recursos adicionales: incluso en aquellas instituciones que contratan profesionales el costo-beneficio sigue siendo mayor. Las tutorías tienen consecuencias directas en la inclusión en educación superior y en la progresión académica de los EsD. Desde la posición de Lissi y Onetto, (2012, citado en Victoriano, 2017), la ayuda de pares y compañeros con actitudes inclusivas permite un aumento de actitudes positivas hacia estas personas, la 
experimentación de sentimientos de éxito, mayores oportunidades de interacción social y el aumento de habilidades académicas.

Finalmente, es importante promover la inclusión en todos los contextos, no solamente en el ámbito educativo, sino también en el cultural o recreacional. Esto permitirá a los estudiantes establecer un sentido de pertenencia hacia su unidad educativa. De acuerdo con lo anterior, se esperaría que, para promocionar y difundir eventos culturales y extraprogramáticos, se entreguen afiches en Braille o se realicen videos en lengua de señas, o se realice el diseño con contraste o en macrotextos, ya que la participación e igualdad de oportunidades no solo debe reflejarse en aspectos como acceso a la información, uso de la tecnología o difusión de actividades, sino también en acciones concretas de participación, en todo ámbito en que los estudiantes se sientan parte de la comunidad educativa y puedan interactuar con ella sin ningún tipo de barrera (Mora y Núñez, 2016).

\section{Conclusión}

De acuerdo con los principales hallazgos expuestos y en concordancia con los objetivos de la investigación, una primera reflexión respecto del cumplimiento de las leyes 20.422/2010 y 21.091/2018 es que existen equipos institucionales que promueven la inclusión desde antes de la promulgación de estas leyes, aunque la mayoría ha conformado sus equipos desde 2007, existiendo algunas instituciones de educación superior que lo han hecho en 2019.

En esta misma línea, no todas tienen un programa de inclusión propiamente tal, algunas contribuyen a mejorar los procesos inclusivos desde los departamentos de bienestar estudiantil y otras lo hacen desde la voluntad de entregar igualdad de oportunidades. Igualmente, la mayoría ha instaurado programas que trabajan en pro de la educación inclusiva, con recursos propios y del Estado, en miras de incorporar políticas inclusivas a los proyectos educativos institucionales.

El discernimiento de lo que acontece en cada institución y el conocimiento de las necesidades reales de la comunidad es una labor 
que recae directamente en los equipos de gestión —rectores, decanos, directores de escuela, jefes de carrera—, quienes, conscientes del rol que desempeñan, deben velar por una comunicación permanente, que recoja inquietudes de sus administrativos, académicos y estudiantes, que permita tomar decisiones con repercusiones positivas en la institución y que beneficie el clima de trabajo y los aprendizajes de los estudiantes (Ministerio de Educación, 2017).

En relación con el acceso al currículo y a las ayudas técnicas, la totalidad de las instituciones de educación superior cuentan con la cantidad suficiente de material de apoyo para el EsD visual, destacando el apoyo brindado por SENADIS a través de fondos adjudicados por medio de proyectos, los que van en directo beneficio de los estudiantes.

Por otra parte, al describir los ítems de matrícula y los registros históricos, se evidencia que no existen datos que permitan analizar la categoría de "eficiencia" en relación con el número de estudiantes que ingresan, desertan y egresan de los planteles educativos, presentándose como un gran desafío a cumplir.

Una de las principales dificultades detectadas en este estudio corresponde a la baja participación de los docentes en las capacitaciones para mejorar las prácticas inclusivas. Esto ha impedido contar con docentes involucrados con la diversidad y, en ocasiones, hasta se han convertido en la barrera más compleja de derribar, ya que, según Salinas, Lissi, Medrano, Zuzulich y Hojas (2013), los prejuicios y las bajas expectativas de los docentes entorpecen los procesos de enseñanza-aprendizaje con EsD. Además, Paz-Maldonado (2020) refiere que una de las principales limitaciones con relación a la inclusión es la falta de competencias de los académicos para atender a EsD.

Finalmente, un factor primordial para hacer efectivo el cambio es que los criterios de inclusión sean parte de la acreditación institucional y la certificación pública por parte de la Comisión Nacional de Acreditación. De este estudio se desprende la necesidad de establecer compromisos institucionales, acordes con los desafíos 
que enfrenta la educación superior para responder a la diversidad. Será un proceso complejo, que favorecerá a las instituciones en pos de la construcción y desarrollo de una cultura inclusiva, destacando el rol fundamental de los docentes en la formación y desarrollo de los estudiantes, y transformando las aulas en espacios dinámicos, diversos, equitativos, vinculantes e inclusivos.

\section{Referencias}

Booth, T. y Ainscow, M. (2015). Guía para la evaluación y mejora de la educación inclusiva. Desarrollando el aprendizaje y la participación en las escuelas. ( $3^{a}$ Edición ed.). España: Centre for Studies on Inclusive Education.

Canales, M. (2006). Metodologías de investigación social. Introducción a los oficios. Santiago de Chile: LOM Ediciones.

Castellanos, A. y Quintana, P. (2019). La inclusión laboral de personas en condición de discapacidad. Espirales Revista Multidisciplinaria de investigación, 3(30), 76-86. DOI: https://doi.org/10.31876/er.v3i30

Castro, L., Antonio Casas, J., Sánchez, S., Vallejos, V. y Zúñiga, D. (2016). Percepción de la calidad de vida en personas con discapacidad y su relación con la educación. Estudios Pedagógicos (Valdivia), 42(2), 39-49. DOI:10.4067/s0718-7052016000200000

DEMRE. (10 de noviembre de 2019). DEMRE Programa de Inclusión. Recuperado de: https://psu.demre.cl/inclusion/index.php

Fajardo, M. S. (2017). La educación superior inclusiva en algunos países de Latinoamérica: avances, obstáculos y retos. Revista Latinoamericana de Educación Inclusiva, 11(1), 171-197. DOI:10.4067/s071873782017000100011

Giraldo, M. (2011). Abordaje de la investigación cualitativa a través de la teoría fundamentada en los datos. La investigación en ingeniería: jugando roles en las ciencias sociales. Venezuela: Actualidad y Nuevas Tendencias.

Guerra, C. M. (2006). Proyectos de integración escolar. Factores que facilitan y obstaculizan su funcionamiento. Santiago: Universidad de Chile.

Lagos, O. (2019). Diseño universal para el aprendizaje: una experiencia innovadora en el aula matemática de octavo año básico. Revista de estudios y experiencias en educación, 18(36), 257-267. DOI: https:// dx.doi.org/10.21703/rexe.20191836lagos3

Ley $N^{\circ}$ 21.091. (29 de mayo de 2018). Sobre educación superior. Diario Oficial de la República de Chile. 
Ley $N^{o}$ 20.422. (10 de febrero de 2010). Establece normas sobre igualdad de oportunidades e inclusión social de personas con discapacidad. Diario Oficial de la República de Chile.

Ley No 20.609, E. m. (02 de mayo de 2012). Ley Chile. Recuperado de www. leychile.cl: http://bcn.cl/luyqt

Lissi, R., Zuzulich, S., Salinas, M., Achiardi, C., Hojas, A. y Pedrals, N. (2009). Discapacidad en contextos universitarios: Experiencia del Piane UC. Calidad en la Educación, (30), 306-324. DOI: https://doi. org/10.31619/caledu.n30.183

Lizama, O., Gil, F. J. y Rahmer, B. (2018). La experiencia de la inclusión en la educación superior en chile ( $2^{\text {a }}$ edición). Santiago de Chile: Universidad de Santiago de Chile.

Ministerio de Educación. (2017). Politicas e iniciativas inclusivas en universidades estatales. División de Educación Superior, MINEDUC. Recuperado de: http://biblioteca.digital.gob.cl/bitstream/handle/123456789/742/ Documento\%20s obre\%20Politicas\%20e\%20Iniciativas\%20dic2017. pdf?sequence $=1$

Mora, M. y Núñez, D. (2017). Inclusión de estudiantes en situación de discapacidad en la Universidad de Chile. Polyphōnía. Revista de Educación Inclusiva, 1(2). Recuperado de: https://revista.celei.cl/ index.php/PREI/article/view/120-140

Otzen, T. y Manterola, C. (2017). Técnicas de muestreo sobre una población a estudio. Int. J. Morphol., 35(1): 227-232.

Paz-Maldonado, E. (2020). Inclusión educativa del alumnado en situación de discapacidad en la educación superior: una revisión sistemática. Teoría de la Educación, 32(1), 123-146. Recuperado de https://dialnet. unirioja.es/servlet/articulo?codigo $=7174727$

RESI. (2018). Red Nacional de Educación Superior Inclusiva. Recuperado de: https://www.resichile.cl

Salinas, M., Lissi, M. R., Medrano, D., Zuzulich, M. D. y Hojas, A. M. (2013). La inclusión en la educación superior: desde la voz de estudiantes chilenos con discapacidad. Revista Iberoamericana de Educación, 63, 77-98. DOI: https://doi.org/10.35362/rie630502

SENADIS. (2016). II Estudio Nacional de la Discapacidad. [Conjunto de datos]. SENADIS. Recuperado de: https://www.senadis.gob.cl/pag/306/1570/ publicaciones

Soler, P. y Fernández, B. (2012). La grounded theory y la investigación cualitativa en comunicación y marketing. ICONO14 Revista Científica 
320 ACCIONES PARA LA IMPLEMENTACIÓN DE PROCESOS INCLUSIVOS EN INSTITUCIONES DE EDUCACIÓN SUPERIOR - Y. Gutiérrez, M. San Martín

de Comunicación y Tecnologías Emergentes, 8(2), 203-213. DOI: https:// doi.org/10.7195/ri14.v8i2.253

UNESCO. (2008). Oficina Internacional de Educación. Recuperado de: http:// www.ibe.unesco.org/es/cie/48areunion-2008.html

Victoriano, E. (2017). Facilitadores y barreras del proceso de inclusión en educación superior: la percepción de los tutores del programa PianeUC. Estudios Pedagógicos (Valdivia), 43(1), 349-369. DOI:10.4067/ s0718-7052017000100020

Recibido: 01/04/2020

Aceptado: 22/10/2020 